\title{
A Comparative Study of the 'rhodochrous' Complex and Related Taxa by Delayed-type Skin Reactions on Guinea Pigs and by Polyacrylamide Gel Electrophoresis
}

\author{
By IRIS S. HYMAN AND SOTIROS D. CHAPARAS \\ Bureau of Biologics, Food and Drug Administration, \\ 8800 Rockville Pike, Bethesda, Maryland 20014, U.S.A.
}

(Received 2 February 1977)

\begin{abstract}
SUMMARY
Cell extracts prepared by ultrasonic disruption of I 7 strains of the 'rhodochrous' complex and related taxa were compared by polyacrylamide gel electrophoresis and, for immunologic relatedness, by skin test reactions. Two organisms, Jensenia canicruria and Nocardia calcarea, gave similar gel patterns and skin test reactions, and are considered to be identical. Extracts of Nocardia rubra showed a strong antigenic relationship with those of three Nocardia pellegrino organisms ( 3225 , $\mathrm{N} 324$ and N420) previously assigned to the 'rhodochrous' complex. Two Gordona organisms appeared to be less antigenically related to the 'rhodochrous' complex. Extracts of three of four organisms designated Lspi (Rhodococcus coprophilus Rowbotham \& Cross 1976) elicited skin test reactions similar to those of the 'rhodochrous' strains. One Lspi strain, N650, showed striking similarities to the 'rhodochrous' complex strain N420 (Nocardia pellegrino).
\end{abstract}

\section{INTRODUCTION}

Nocardioforms are diverse organisms, varying in their biochemical, morphological and nutritional requirements, but they all form a primary mycelium which fragments into rods and cocci. Many of these bacteria have been classified in the genera Nocardia, Mycobacterium or in the 'rhodochrous' complex (Cross \& Goodfellow, 1973). Recently, methods for phage typing, determination of wall composition and lipid content, and immunological studies have been explored to identify good diagnostic characters. In a numerical taxonomic study, Goodfellow (I97I) classified 283 nocardioform bacteria into I5 clusters, one of which was equated with the 'rhodochrous' complex (Goodfellow, I97I ; Goodfellow et al., 1974). In the present studies, extracts of I 4 selected organisms of the 'rhodochrous' complex and three other species were analysed for relatedness by their reactivities in skin testing on sensitized guinea pigs and by polyacrylamide gel electrophoresis (PAGE).

\section{METHODS}

Strains. The test strains were from Dr M. Goodfellow, University of Newcastle upon Tyne (Table I).

Cultures. Cultures were grown on oleic acid albumin and Lowenstein-Jensen egg yolk slants for 2 to 4 days, then inoculated into Blake bottles containing $200 \mathrm{ml}$ of Long's synthetic medium (Darzins, 1958; Drea \& Andrejew, 1953). Most strains were harvested within a 
Table I. Species tested (clusters are those designated by Goodfellow, I97I)

\begin{tabular}{|c|c|}
\hline \multirow[b]{2}{*}{ N324 } & \\
\hline & $\begin{array}{l}\text { 'rhodochrous' strain (Nocardia pellegrino): G. Castelnuova, Istituto Superiore Di Sanita, } \\
\text { Rome, Italy; ATCCI } 5998 \\
\text { 'rhodochrous' strain (Nocardia pellegrino); G. Castelnuova, 906B } \\
\text { 'rhodochrous' strain (Nocardia pellegrino); A. Tacquet, University of Lille, France, } 906\end{array}$ \\
\hline & Cluster 14 (subgroup $14 \mathrm{C}$ ) \\
\hline $\begin{array}{l}\text { N5 } \\
\text { N54 }\end{array}$ & $\begin{array}{l}\text { 'rhodochrous' strain (Nocardia salmonicolor); NCIB9701 } \\
\text { 'rhodochrous' strain (Mycobacterium rhodochrous); ATCCI3808; R. E. Gordon, Rutgers } \\
\text { University, U.S.A., 372; (Rhodococcus rhodochrous) }\end{array}$ \\
\hline & Cluster I4 (subgroup 14D) \\
\hline $\begin{array}{l}\text { N38 } \\
\text { N53 }\end{array}$ & $\begin{array}{l}\text { 'rhodochrous' strain (Nocardia opaca); NCIB9409 } \\
\text { 'rhodochrous' strain (Jensenia canicruria); NCIB8I47; ATCCI I048 }\end{array}$ \\
\hline & Cluster I4 (subgroup I4E) \\
\hline NI 24 & 'rhodochrous' strain (Nocardia opaca); CBS330.6I \\
\hline & Cluster I (subgroup IA) \\
\hline N99 & Nocardia asteroides; R. E. Gordon, 508 \\
\hline & Other strains \\
\hline $\begin{array}{l}\text { N4I } \\
\text { N36I }\end{array}$ & $\begin{array}{l}\text { 'rhodochrous' strain (Nocardia calcarea); } \mathrm{NCIB8863} \\
\text { 'rhodochrous' strain (Nocardia rubra); M. Tsukamura, National Chubu Hospital, Obu, Aichi } \\
\text { 474, Japan, M-I }\end{array}$ \\
\hline $\begin{array}{l}\text { N655 } \\
\text { N656 } \\
\text { N650 to N653 }\end{array}$ & $\begin{array}{l}\text { Gordona rubra; NCTCIo668; M. Tsukamura, } 3605 \\
\text { Gordona terrae; NCTClo669; M. Tsukamura, } 3623 \\
\text { Lspi strains; T. J. Rowbotham, University of Bradford, CuB } 628 \text {, I I8, 670, } 669\end{array}$ \\
\hline
\end{tabular}

few days, but N656, N54 (Mycobacterium rhodochrous) and the Lspi strains, N650 to N653, required I to 2 weeks incubation. Although Lspi strains are reported to require thiamin for growth (Rowbotham \& Cross, 1976), these strains, as well as N54, grew abundantly when the inoculum size and incubation period were increased. The same medium for all cultures was preferred in order to minimize variables.

Preparation of antigens. Organisms were washed three times with sterile distilled water by centrifugation at $2000 \mathrm{~g}$ at $4{ }^{\circ} \mathrm{C}$ for $\mathrm{I} 5 \mathrm{~min}$. A portion of the packed organisms was dried on a watch glass at $35^{\circ} \mathrm{C}$ and used for sensitizing guinea pigs; the remainder was disrupted ultrasonically and used for skin testing and for polyacrylamide gel electrophoresis. Disruption was achieved by treating a $50 \%(\mathrm{w} / \mathrm{v})$ suspension of packed organisms in sterile distilled water for 5 to 10 min at maximum output in a Bronson W-350 cell disruptor cooled with circulating ice water. The extracts were then centrifuged for 20 to $30 \mathrm{~min}$ at $26000 \mathrm{~g}$ at $4{ }^{\circ} \mathrm{C}$. The supernatant fluid was sterilized by passage through a $0.2 \mu \mathrm{m}$ Nalgene filter and stored in $3 \mathrm{ml}$ portions at $-20^{\circ} \mathrm{C}$.

Sensitization and skin testing of guinea pigs. Groups of eight Hartley strain female guinea pigs weighing between 300 and $400 \mathrm{~g}$ were injected subcutaneously in the nuchal area with $0.2 \mathrm{ml}$ of an emulsion containing $2 \mathrm{mg}$ dried organisms. The emulsions were made with Arlacel A (Atlas Chemicals)/Drakeol 6VR (Penreco)/saline (35:65:100, by vol.). Six weeks after sensitization, the activity of the extracts was screened in four of the sensitized guinea pigs by skin testing with $0.1 \mathrm{ml}$ saline containing various amounts of the homologous antigen. Skin reactions were read after $24 \mathrm{~h}$. A dose was selected which produced an erythmatous reaction of approximately $\mathrm{I} 3 \mathrm{~mm}$ diam. This dose, determined for each of the homologous systems, will be referred to as the bioequivalent dose.

The eight guinea pigs in each group, including a group of control animals which had not 
previously been sensitized, were then skin tested with $0.1 \mathrm{ml}$ of each of the bioequivalent skin test doses. Since the four animals which had been used to determine each bioequivalent skin test dose reacted in the same way as those not previously tested, the results were pooled and analysed according to Magnusson's procedure for specificity differences (Magnusson, Engbaek \& Bentzon, 196r; Magnusson, 197I, 1973).

Polyacrylamide gel electrophoresis. Electrophoresis was performed as described by Davis (I964), using the Canalco Disc Electrophoresis apparatus, model I 200 (5635 Fishers Lane, Rockville, Maryland 20852, U.S.A.) with $75 \mathrm{~mm}$ long gel tubes at $5 \mathrm{~mA}$ per tube for 35 to $40 \mathrm{~min}$. The separating gel contained acrylamide $(7 \%, \mathrm{w} / \mathrm{v})$ at $\mathrm{pH} 8.9$ and the stacking gel contained acrylamide $(2 \cdot 5 \%, \mathrm{w} / \mathrm{v})$ at $\mathrm{pH} 6.7$ (Davis, 1964$)$. Gel patterns were compared by measuring the relative mobilities of prominent bands.

\section{RESULTS AND DISCUSSION}

The mean homologous and heterologous skin test reactions were determined for the extracts of the 17 strains at the bioequivalent dose (Table 2). The results of skin tests for all the control animals were negative. Some heterologous antigens gave an equal or slightly greater reaction than that given by the homologous antigen.

The specificity differences (SPD) between each of the 17 organisms were analysed according to Magnusson's method (Table 3). A small SPD indicates a close relationship between strains and several groups have been indicated (groups I to III).

One striking finding was that rhodochrous strain N324 ( $N$. pellegrino) cross-reacted extensively with all rhodochrous and Lspi strains, as indicated by the low SPD values, but not with $N$. asteroides or the Gordona species. Thus, N324 was a good indicator strain for unifying the rhodochrous and Lspi strains, as well as closely related strains. Another interesting result was that type strain N54 ( $M$. rhodochrous) had low SPD values with all Lspi strains, including N652.

Gels with similar PAGE patterns have been grouped together in Fig. I. Each group (I to IV) includes gels with corresponding band numbers, as discussed below.

Strains from subgroup I4D, which includes N53 (Jensenia canicruria) and N38 (Nocardia opaca), have been linked above the $90 \% \mathrm{~S}$ (similarity) level (Goodfellow, I97I) and were very similar to N4I (Nocardia calcarea). To facilitate comparison of bands in PAGE patterns of these three organisms, the corresponding prominent bands in each gel have been numbered (Fig. I, group III). Although the rates of migration of the bands varied among some of the preparations, the similarities of the numbered bands were confirmed by measurements of relative mobilities (Table 4 ). (Some of the labelled bands are not as distinct in the photograph as they were in the actual gel.) Strains N38 (N. opaca) and N53 (J. canicruria) shared at least 12 bands ( 3 and 7 to 17 ), a finding consistent with the reported numerical phenetic relationship (Goodfellow, 197I) and with the low SPD value of 0.54. Strains N4I (N. calcarea) and N53 (J. canicruria) also shared a low SPD value of o.38 (Table 3 ) and the relative mobilities of all discernible bands were identical (Table 4). The findings, therefore, strongly suggest that $N$. calcarea should be classified in subgroup I4D (Goodfellow, I97I).

Representative strains from subgroup I4A, which includes rhodochrous strains N324, N325 and N420, have been grouped at the 95\% S-level (Goodfellow, 197I). The PAGE patterns (Fig. I) support this close relationship, and also suggest that strain N36I (Nocardia rubra) should be classified in subgroup I $4 \mathrm{~A}$. There were at least 1o bands $\left(3\right.$ to $8,12,1_{3}, 1_{5}$ and 17 ) shared by each of the four organisms (Table 4). An especially close resemblance was seen between $\mathrm{N} 325$ and N420 which both displayed the additional bands I, 2, 9, I4 and 


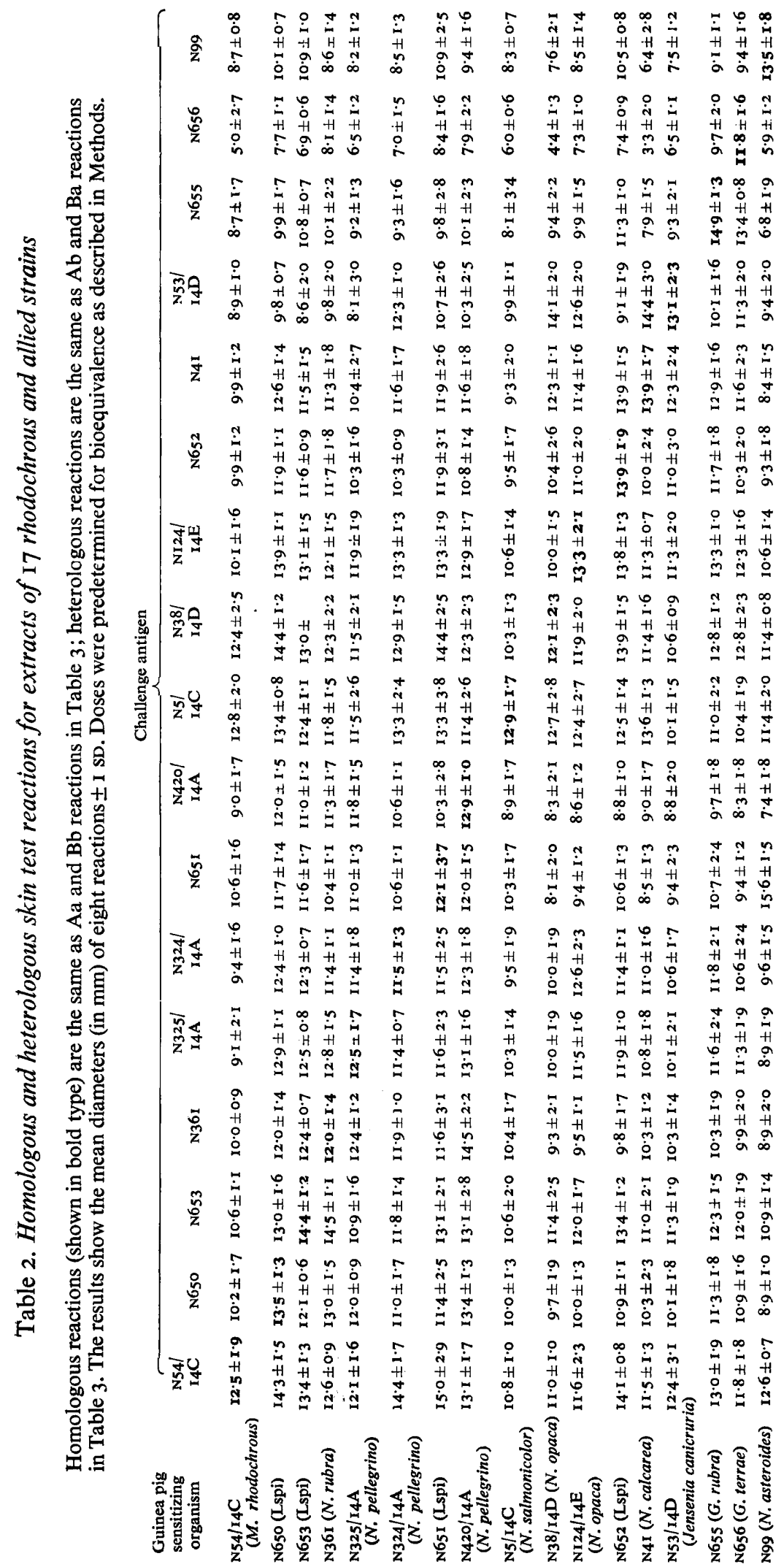


Table 3. Specificity differences (SPD) of antigens of 17 rhodochrous and allied strains

Specificity difference (SPD) is defined as $(A a+B b)-(A b+B a)$, in which $A a$ and $B b$ are the diameters of the homologous $\mathrm{A}$ and $\mathrm{B}$ reactions respectively, and $\mathrm{Ab}$ and $\mathrm{Ba}$ are the diameters of the heterologous $A$ and $B$ reactions respectively. $A$ and $B$ indicate guinea pigs sensitized with strain $A$ and strain B emulsions respectively. For details, see Magnusson et al. (I96I) and Magnusson (I97I, 1973).

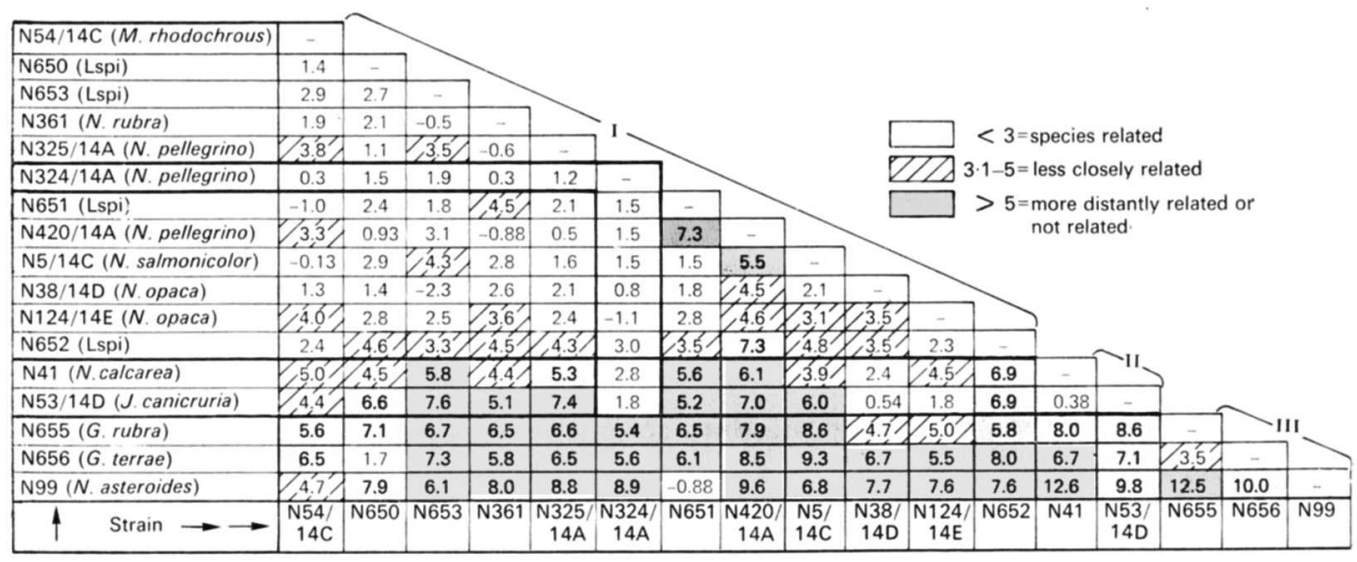

I6. The close relationship between the four strains was reinforced by the SPD values which were all less than $I^{\cdot} 5$ (Table 3 ).

Subgroup I4C included strains N5 (Nocardia salmonicolor) and N54 (M. rhodochrous), which have been linked above the $90 \%$ S-level (Goodfellow, I97I). The SPD value of 0.13 supports a close relationship between the two organisms, although the affinity was not as obvious in the disc gel patterns (Fig. I, group II). The extract of strain N54 gave more bands than that of strain N5, but at least eight corresponding bands were discerned.

The only representative of subgroup I4E studied was strain NI24 (N. opaca) which was most closely related to strains $\mathrm{N} 324$ ( $N$. pellegrino) in I4A, and N53 (Jensenia canicruria) in I4D, with SPD values of less than 2 (Table 3). Comparisons of the PAGE patterns of strains NI24 and N324 (Fig. I, group I) and $R_{F}$ values (Table 4) indicate a similarity in at least nine bands, while $R_{F}$ values (not all shown in Table 4) for strains NI 24/14 E and N53/I4D suggest that four bands correspond ( $0.10,0.45,0.49$ and 0.56$)$.

Three of the four Lspi strains, N650, N65I and N653, shared relatively low SPD values (I 8 to $2 \cdot 7$ ). Strains N650 and N653 shared the most bands (Table 4). Strains N65I and N653 shared the lowest SPD value of $\mathrm{I} \cdot 8$, and the $R_{F}$ values of five of their bands were similar (Table 4). The fourth Lspi strain, N652, shared a somewhat higher SPD value (3.3 to $4 \cdot 6$ ) with each of the other three strains. Strain $\mathrm{N} 652$ had three bands with similar $R_{F}$ values to N65I and five bands similar to those of N650. Strain N650 fits well in group I (Table 3) with strains of subgroup I4A, N38/14D (N. opaca) and Lspi strains N65I and N653. An SPD value of 0.93 between $\mathrm{N} 650$ and rhodochrous strain $\mathrm{N} 420(N$. pellegrino) suggested a close antigenic relationship. The PAGE patterns of these two strains were similar (Fig. I, group I) and there were at least 15 corresponding bands (Table 4). Strain N653 (Lspi) shared an SPD value of 0.5 with strain N36I (N. rubra) and their PAGE patterns had seven corresponding bands. Strain N65I (Lspi) shared low SPD values with strains N99 ( $N$. asteroides) from cluster I $(-0 \cdot 88)$, and N5 $(N$. salmonicolor) $(\mathrm{I} \cdot 5)$ and N54 $(M$. rhodochrous $(-\mathrm{I} \cdot 0)$ from subgroup I4C (Goodfellow, I97I). $R_{F}$ values indicate possible identity of seven bands of strains N65I 


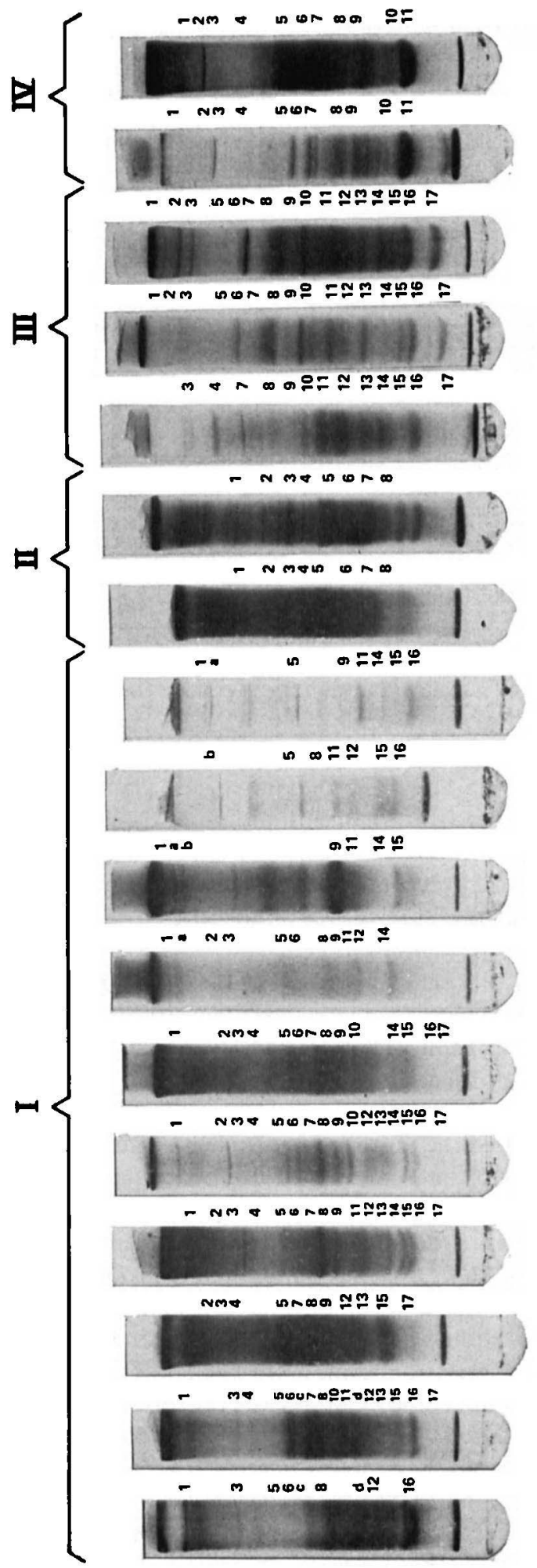

Fig. I. PAGE patterns of 17 rhodochrous and allied strains, indicating similarities in band patterns. Identification of the more prominent bands by number is given to the right of each band. Groups I to IV indicate gels with an identical numbering system. 


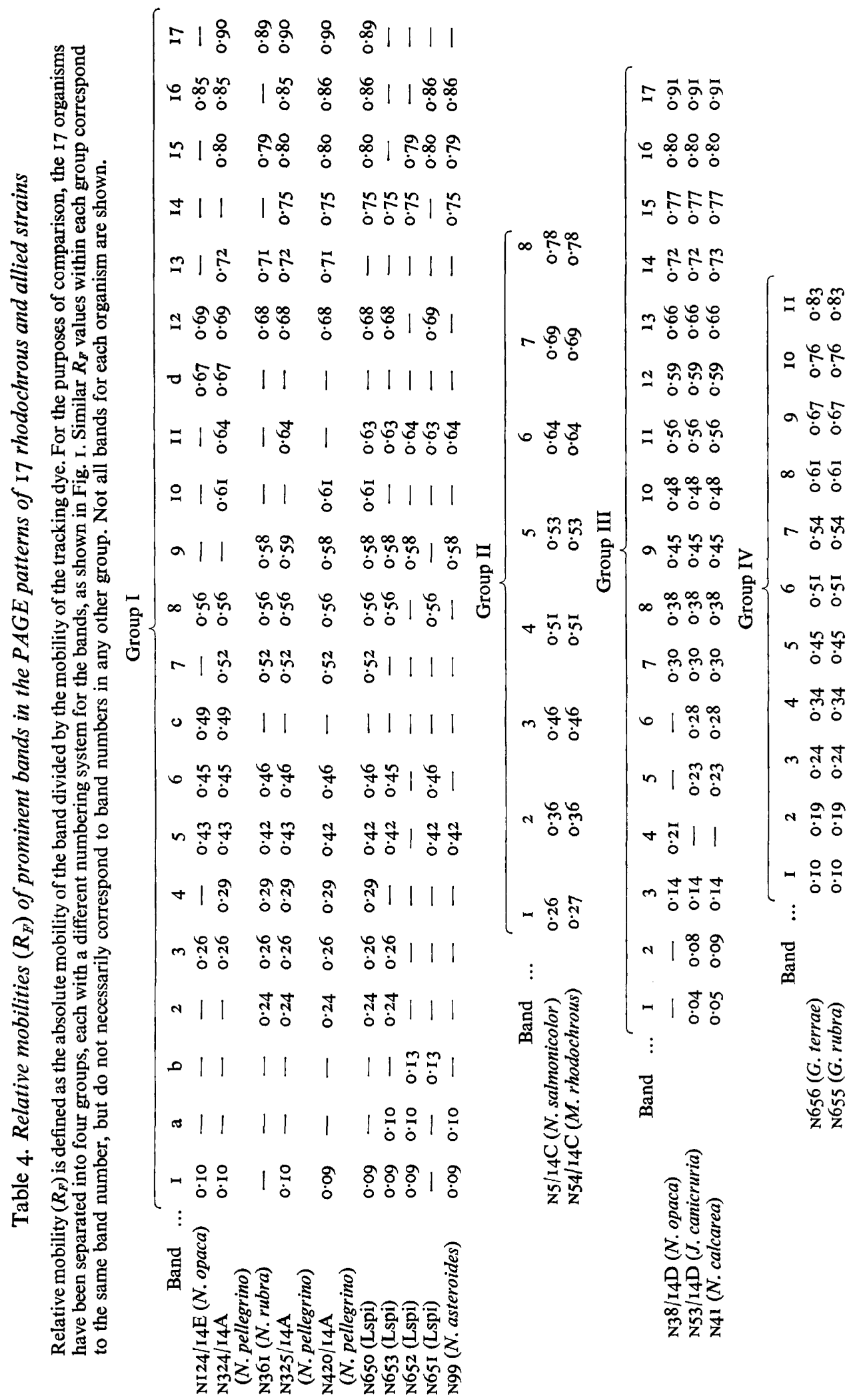


and N5 $(0.38,0.42,0.46,0.5 \mathrm{I}, 0.63,0.64$ and 0.69$)$, and six bands of strains N65I and N54 (0.1 I, 0.46, 0.5I, 0.56, 0.63 and 0.69). Not all of the $R_{F}$ values are shown in Table 4.

Strain N99 ( $N$. asteroides), except for a small SPD shared with strain N65I, was not closely related to any other strain. SPD values ranged from 4.7 to $12 \cdot 6$, with an average of 8.5 , which supports the suggestion that the 'rhodochrous' complex is distinct from the Nocardia group (Goodfellow et al., 1974). The seeming discrepancy in the low SPD value between two distantly related organisms such as strains N99 and N65I is probably due to dominant skin reactive antigens shared by both species. Each skin reaction is the summation of the interaction between a number of antigens interacting with specifically sensitized lymphocytes. When two organisms share a large number of antigens, the relationship usually results in a low SPD value. Occasionally, as in the present instance, organisms which are apparently distantly related share a low SPD. If such organisms share a few antigens towards which sensitivity may be strong and predominant, this could result in the induction of mutually large skin test reactions and a low SPD. Although only four antigens were matched between strains N99 and N65I, these may be predominant shared antigens that account for the skin reactivity. However, other antigens may be involved even though they are not matched by PAGE patterns. Confirmation of this suggestion can only be made by isolating such antigens and by demonstrating their antigenic similarity. The Weil-Felix reaction (Davis et al., 1969) is an example of similar antigens being produced by two distantly related organisms, the Rickettsia and Proteus species.

Organisms of the genus Gordona were originally isolated from sputa of patients with pulmonary disease and from soil. Biochemical, nutritional and morphological tests and the degree of acid fastness provided criteria by which the gordonae were placed between the genera Nocardia and Mycobacterium. Gordona species were found to be most closely related to mycobacteria, differing in only one or two characteristics, and Tsukamura (I971) proposed that certain $M$. rhodochrous strains be classified in the genus Gordona. Our findings do not support this proposal. The specificity differences with the rest of the complex were high overall (Table 3), with the exception of strain N656 (Gordona terrae) and Lspi strain N650, and suggested that the two Gordona species tested are distinct and share few antigenic determinants with the rhodochrous organisms. Gordona and rhodochrous strains have also been distinguished on the basis of differences in their mycolic acids (Alshamaony, Goodfellow \& Minnikin, 1976) and antibiotic sensitivity patterns (Goodfellow \& Orchard, 1974). The relatedness of the two Gordona species was supported by the PAGE patterns, which indicated at least I I corresponding bands (Fig. I, group IV).

The taxonomic structure of the 'rhodochrous' complex is emerging. Relatedness among organisms has largely been determined by morphology, biochemical activity, temperature requirements and cultural appearance. Various immunological techniques have been found to be of taxonomic value at the generic, subgeneric, subspecific and infraspecific levels (Runyon, 1959; Schaefer, 1965; Stanford \& Beck, 1968; Magnusson, 1973; Stanford \& Grange, 1974). In the present studies, antigenic relatedness was assessed by the ability of extracts to induce skin reactions in sensitized guinea pigs. The results can be used to support some earlier groupings which had been proposed on the basis of biochemical, nutritional and morphological criteria. The PAGE patterns, although also not specific, offered a preliminary glimpse of presumptive antigenic identity. Since every protein has the potential of being an antigen, it is not unreasonable to assume that protein staining bands are antigens, or at least haptens. When two or more isolates shared specificity differences of $I$ or less, there was good correlation with the PAGE studies in which similar patterns in appearance and band migration were observed. Studies are currently in progress to define more accurately, and with specific antisera, the degree of relatedness within this complex group. 


\section{REFERENCES}

Alshamaony, L., Goodfellow, M. \& Minnikin, D. E. (1976). Free mycolic acids in the classification of Gordona and the 'rhodochrous' complex. Journal of General Microbiology 92, $183-187$.

CROss, T. \& GoOdfellow, M. (1973). Taxonomy and classification of the actinomycetes. In Actinomycetales: Characteristics and Practical Importance, pp. I I-I I2. Edited by G. Sykes and F. A. Skinner. London: Academic Press.

Darzins, E. (1958). The Bacteriology of Tuberculosis, pp. 213-228. London: Oxford University Press.

DAvis, B. J. (1964). Dise electrophoresis. II. Background and theory. Annals of the New York Academy of Sciences I21, 404-427.

Davis, B., DulbeCCO, R., EISEN, H., GinsBeRG, S. \& Wood, B. (I969). Microbiology, pp. 928-946. New York: Harper \& Row.

Drea, W. F. \& ANDREJew, I. C. (1953). The Metabolism of the Tubercule Bacillus, pp. 67-r18. Springfield, Illinois: Charles C. Thomas.

Goodfellow, M. (1971). Numerical taxonomy of some nocardioform bacteria. Journal of General Microbiology $69,33-80$.

Goodfellow, M. \& ORCHARD, V. A. (1974). Antibiotic sensitivity of some nocardioform bacteria and its value as a criterion for taxonomy. Journal of General Microbiology 83, 375-387.

Goodfellow, M., Lind, A., Mordarska, H., PatTyn, S. \& TsuKamura, M. (I974). A co-operative numerical analysis of cultures considered to belong to the 'rhodochrous' taxon. Journal of General Microbiology $85,291-302$.

Magnusson, M. (1971). A comparative study of Mycobacterium gastri and Mycobacterium kansasii by delayed type skin reactions in guinea pigs. American Review of Respiratory Diseases 104, 377-384.

Magnusson, M. (1973). Taxonomic usefulness of skin tests on guinea pigs with sensitins of mycobacteria. In Atypical Mycobacteria, pp. 23-26. Edited by J. G. Weiszfeiler. Budapest: Akadémiai Kiadó.

Magnusson, M., EngBaek, H. E. \& Bentzon, M. W. (1961). Specificity of mycobacterial sensitins. American Review of Respiratory Disease 83, 57-68.

Rowbotham, T. J. \& Cross, T. (I976). Rhodochrous-type organisms from freshwater habitats. In Proceedings of the Society for General Microbiology 3, roo-ror.

RuNYON, E. H. (1959). The differentiation of anonymous (atypical) mycobacteria in mammalian tubercle bacilli. Bulletin of the International Union on Tuberculosis 29, 69-78.

SCHAEFER, W. B. (1965). Serologic identification and classification of the atypical mycobacteria by their agglutination. American Review of Respiratory Diseases 92, 85-93.

StANFORD, J. L. \& BeCK, A. (1968). An antigenic analysis of the mycobacteria, Mycobacterium fortuitum, M. kansasii, M. phlei, M. smegmatis and M. tuberculosis. Journal of Pathology and Bacteriology 95, I3I-I39.

Stanford, J. L. \& Grange, J. M. (1974). The meaning and structure of species as applied to mycobacteria. Tubercle 55, 143-152.

TsukamuRA, M. (1971). Proposal of a new genus, Gordona, for slightly acid-fast organisms occurring in sputa of patients with pulmonary disease and in soil. Journal of General Microbiology 68, 15-26. 